\title{
CONHECIMENTO MATEMÁTICO PARA O ENSINO (MKT): UM LEVANTAMENTO BIBLIOGRÁFICO EM DISSERTAÇõES E TESES BRASILEIRAS
}

\author{
MATHEMATICAL KNOWLEDGE FOR TEACHING (MKT): A BIBLIOGRAPHIC \\ SURVEY IN BRAZILIAN DISSERTATIONS AND THESES
}

DOI: 10.23926/RPD.2526-2149.2020.v5.n2.p608-625.id689

\author{
André Lima \\ Rodrigues \\ Menstrando em Ensino de \\ Ciências e Educação \\ Matemática (UEL) \\ andrelr1@hotmail.com
}

\section{Bruno Rodrigo \\ Teixeira}

Doutor em Ensino de

Ciências e Educação

Matemática (UEL)

Docente do Departamento de Matemática da Universidade Estadual de Londrina (UEL) bruno@uel.br
Resumo: Este estudo tem por objetivo identificar a partir de quais elementos o Conhecimento Matemático para o Ensino (MKT) tem sido investigado e ou identificar os elementos que têm sido analisados com base no MKT em dissertações e teses brasileiras, por meio de um levantamento bibliográfico em dois bancos de dados e da análise dos resumos dos trabalhos. Os resultados indicam que o Conhecimento Matemático para o Ensino tem sido investigado a partir de, ou utilizado para análise de reflexões, compreensões, concepções; ações formativas e práticas profissionais. Além disso, identificamos trabalhos que investigam o MKT, a partir de pesquisas bibliográficas e trabalhos, em que os documentos oficiais foram utilizados para analisá-lo ou como base para a investigação. Os resultados podem orientar formadores de professores quanto a estratégias formativas para o desenvolvimento ou a mobilização de conhecimentos profissionais e pesquisadores para investigações futuras.

Palavras-chave: Educação Matemática. Formação de Professores. Conhecimentos profissionais docentes. Conhecimento Matemático para o Ensino. MKT.

\begin{abstract}
This study aims to identify from which elements the Mathematical Knowledge for Teaching (MKT) has been investigated and or identify the elements that have been analyzed based on MKT in Brazilian dissertations and theses, through a bibliographic survey in two databases and an analysis of the works' abstracts. The results indicate that the Mathematical Knowledge for Teaching has been investigated from, or used to analyze, reflections, understandings, conceptions; formative actions and professional practices. Furthermore, we identified works that investigate MKT from bibliographic searches and works in which official documents were used either to analyze it or as a basis for the investigation. The results can guide teacher-trainers as regards to formation strategies to the development or the mobilizations of professional knowledge and researchers for future investigations.

Keywords: Mathematics Education. Teacher education. Teacher professional knowledge. Mathematical Knowledge for Teaching. MKT.
\end{abstract}




\section{INTRODUÇÃO}

Diversos estudos mostram que aos professores é necessário mais do que o conhecimento do conteúdo da disciplina que lecionam e que eles mobilizam um conjunto de conhecimentos especializados (GARCIA, 2014).

Entre esses estudos pode ser destacado o de Shulman (1986), que propõe um modelo muito utilizado em pesquisas e como fundamento teórico na preparação docente (MORIEL JUNIOR; WIELEWISCK, 2017). Nele, o autor organiza o conhecimento profissional docente em três categorias, nomeadamente: Conhecimento do Conteúdo, Conhecimento Curricular e Conhecimento Pedagógico do Conteúdo, sistematizando-o a partir da descrição das características de cada categoria.

Entretanto, por ser um modelo genérico, possui limitações para discussões específicas relacionadas a uma disciplina, como a Biologia, a Matemática ou a Física (MORIEL JUNIOR; WIELEWISCK, 2017). Diante disso, Deborah Ball e colaboradores propuseram um modelo específico para a Matemática, o Conhecimento Matemático para o Ensino - MKT ${ }^{1}$ (BALL; THAMES; PHELPS, 2008) como um refinamento das categorias do conhecimento estabelecidas por Shulman (1986).

A respeito do MKT, pode-se destacar o seu pioneirismo em descrever o conhecimento de professores de Matemática pelo fato de evidenciar "o papel do conteúdo matemático, ao mesmo tempo em que considera aspectos relacionados ao processo de ensino, como a aprendizagem dos alunos, o currículo, dentre outros" (MORIEL JUNIOR; WIELEWISCK, 2017, p. 129), e sua relevância para a Educação Matemática.

Diante disso, por meio de um levantamento bibliográfico em dissertações e teses brasileiras que utilizam esse modelo, objetivamos com este trabalho identificar a partir de quais elementos o Conhecimento Matemático para o Ensino tem sido investigado e ou identificar os elementos que têm sido analisados com base no MKT. Para isso, apresentamos, a seguir, uma breve fundamentação teórica a respeito dos conhecimentos profissionais docentes na perspectiva do MKT, os aspectos metodológicos e uma seção de resultados e análises seguida de algumas considerações sobre o tema.

\section{REFERENCIAL TEÓRICO}

\footnotetext{
${ }^{1}$ Mathematical Knowledge for Teaching. 
Aspecto fundamental para a formação de professores, o conhecimento profissional docente tem sido objeto de estudo e discussão nas últimas décadas (GARCIA, 2014). O trabalho de Shulman (1986), conforme já destacado anteriormente, tem sido muito difundido e apresentou, entre outras categorias, o chamado conhecimento pedagógico do conteúdo.

Entretanto, segundo Ball, Thames e Phelps (2008),

[...] quase um terço dos artigos que citam o conhecimento pedagógico do conteúdo o fazem sem atenção direta a uma área de conteúdo específica, em vez disso, fazem afirmações gerais sobre o conhecimento do professor, a formação de professores ou a política. Os estudiosos usaram o conceito de conhecimento pedagógico do conteúdo como se seus fundamentos teóricos, distinções conceituais e testes empíricos já estivessem bem definidos e entendidos universalmente (p. 394, tradução nossa).

Diante disso, refinando a categorização, proposta por Lee Shulman (1986), para o conhecimento profissional do professor, Ball, Thames e Phelps (2008) propuseram o modelo denominado Conhecimento Matemático para o Ensino, MKT.

Por 'Conhecimento Matemático para o Ensino', entendemos o conhecimento matemático necessário para realizar o trabalho de ensinar Matemática. É importante observar, aqui, que a nossa definição começa com o ensino, não com os professores. Preocupa-se com as tarefas envolvidas no ensino e as exigências matemáticas dessas tarefas (p. 395, tradução nossa).

A elaboração desse modelo foi apoiada em análises de vídeos da prática do professor (BALL; THAMES; PHELPS, 2008). Nele, o conhecimento profissional do professor de Matemática é posto em dois domínios: o Conhecimento Específico do Conteúdo, subdividido em Conhecimento Comum do Conteúdo, Conhecimento Especializado do Conteúdo e Conhecimento do Conteúdo no Horizonte; e o Conhecimento Pedagógico do Conteúdo, subdividido em Conhecimento do Conteúdo e Estudantes, Conhecimento do Conteúdo e Ensino e Conhecimento do Currículo. A seguir apresentamos uma síntese de cada um deles.

- $\quad$ No Conhecimento Comum do Conteúdo inclui-se o conhecimento não exclusivo ao ensino, "o conhecimento e a habilidade matemática usados em cenários diferentes do ensino" (BALL; THAMES; PHELPS, 2008, p. 399, tradução nossa).

- Conhecimento Especializado do Conteúdo envolve o conhecimento necessário para o ato de ensinar, ou seja, "é o conhecimento e a habilidade matemática únicos para o ensino. [...] é um conhecimento matemático que tipicamente não é necessário para outros propósitos que não seja o ensino.” (BALL; THAMES; PHELPS, 2008, p. 400, tradução nossa). 
- Conhecimento do Conteúdo no Horizonte "é uma consciência de como os tópicos matemáticos estão relacionados ao longo da extensão da Matemática incluída no currículo" (BALL; THAMES; PHELPS, p. 403, tradução nossa).

- Conhecimento do Conteúdo e dos Estudantes engloba a antecipação do que os alunos provavelmente pensam e o que acharão confuso, dos tipos de tarefas que acharão difícil ou não, de como completar um pensamento incompleto de um aluno. Em geral, "é um conhecimento que combina conhecer sobre estudantes e conhecer sobre Matemática" (BALL; THAMES; PHELPS, 2008, p. 401, tradução nossa).

- $\quad$ Conhecimento do Conteúdo e do Ensino: envolve as noções de quais exemplos utilizar para introduzir um conteúdo, potencialidades de abordagens de ensino para a aprendizagem de determinado conteúdo e, no geral, "combina conhecer sobre ensino e conhecer sobre matemática" (BALL; THAMES; PHELPS, 2008, p. 401, tradução nossa).

- Quanto ao Conhecimento do Currículo, trata-se do mesmo conhecimento curricular proposto por Shulman (1986), que envolve

[...] a capacidade do professor de relacionar o conteúdo de um determinado curso ou lição a tópicos ou questões que estão sendo discutidos simultaneamente em outras aulas. [...] a familiaridade com os tópicos e assuntos que foram e serão ensinados na mesma área durante os anos anteriores e posteriores na escola, e os materiais que os incorporam. (SHULMAN, 1986, p.10, tradução nossa).

Diante das potencialidades do MKT para discussões e investigações a respeito dos conhecimentos profissionais de docentes em formação inicial e continuada, bem como para a pesquisa em Educação Matemática, consideramos importante, para a compreensão do que se tem pesquisado a respeito dele, identificar a partir de quais elementos esse conhecimento tem sido investigado e ou identificar os elementos que têm sido analisados com base no MKT. Desse modo, apresentamos, a seguir, nossos delineamentos metodológicos que mostram a trajetória deste estudo para atingir tal objetivo.

\section{Aspectos Metodológicos}

Este estudo foi desenvolvido sob uma abordagem qualitativa (BOGDAN; BIKLEN, 1994), de cunho interpretativo. Inicialmente, realizamos um levantamento bibliográfico em dois bancos de dados, o Catálogo de Dissertações e Teses da CAPES (Coordenadoria de Aperfeiçoamento de Pessoal de Nível Superior $)^{2}$ e a Biblioteca Digital Brasileira de Teses e

\footnotetext{
${ }^{2}$ https://catalogodeteses.capes.gov.br/catalogo-teses/\#!!. A busca foi realizada em 19/06/2019.
} 
Dissertações $(\mathrm{BDTD})^{3}$, com os disparadores "conhecimento matemático para o ensino" e "MKT", separadamente, tendo em vista que alguns autores podem optar por utilizar a sigla em inglês. Como resultado, encontramos 99 trabalhos que foram comparados a fim de retirarmos os que se repetiam.

Esse primeiro movimento permitiu a retirada de 17 trabalhos, estabelecendo um total de 82. Posteriormente, realizamos outros dois movimentos. O primeiro foi a leitura dos títulos, que nos possibilitou retirar 45 trabalhos de outras áreas, como marketing e saúde, e o segundo, a leitura dos resumos, que possibilitou a retirada daqueles que não apresentavam indícios de que utilizariam o Conhecimento Matemático para o Ensino em sua investigação, um total de 11 textos. Com isso, nosso conjunto de trabalhos que seriam submetidos à análise ficou com 26 textos, sendo 15 dissertações e 11 teses.

Para efeitos de análise, todos os textos receberam um código constituído de $\mathrm{D}$, para dissertações, ou de $\mathrm{T}$, para teses, seguido de um número, que indica sua posição na ordem cronológica de defesa, da sigla da instituição em que a pesquisa foi realizada e do ano de defesa. Assim, o código D01UNIBAN2012 se refere à dissertação de número um, defendida na Universidade Bandeirante de São Paulo em 2012.

A seguir, apresentamos as dissertações e teses selecionadas e os códigos a elas atribuídos:

Quadro 1 - Dissertações e teses consideradas para análise

\begin{tabular}{|l|c|}
\hline \multicolumn{1}{|c|}{ TRABALHO } & CÓDIGO \\
\hline $\begin{array}{l}\text { Formação continuada e ensino de álgebra: reflexões de professores da } \\
\text { Educação Básica sobre itens do SARESP. Rosangela de Souza Jorge Ando. } \\
\text { Dissertação de mestrado acadêmico. 2012. }\end{array}$ & D01UNIBAN ${ }^{4} 2012$ \\
\hline $\begin{array}{l}\text { Analisando a mobilização de conhecimentos algébricos de professores de } \\
\text { Educação Básica: o momento de preparação de aulas sobre equações. Felipe } \\
\text { Augusto Pereira Vasconcelos Santos e Oliveira. Dissertação de mestrado } \\
\text { acadêmico. 2014. }\end{array}$ & D02UFABC 2014 \\
\hline $\begin{array}{l}\text { Investigando como professores dos Anos Iniciais julgam propostas de ensino } \\
\text { para o trabalho com números racionais. Josué Ferreira dos Santos Filho. } \\
\text { Dissertação de mestrado acadêmico. 2015. }\end{array}$ & D03UFPE6 2015 \\
\hline $\begin{array}{l}\text { O conceito de simetria na álgebra escolar: um estudo baseado na análise de } \\
\text { documentos oficiais e manuais de professores. Bárbara Passadore de } \\
\text { Oliveira. Dissertação de mestrado acadêmico. 2015. }\end{array}$ & D04UFABC2015 \\
\hline $\begin{array}{l}\text { A formação do professor de Matemática: um estudo sobre o conhecimento } \\
\text { pedagógico dos números racionais. Debora da Silva Souza. Dissertação de } \\
\text { mestrado acadêmico. 2015. }\end{array}$ & D05UFABC2015 \\
\hline
\end{tabular}

\footnotetext{
${ }^{3}$ http://bdtd.ibict.br/vufind/. A busca foi realizada em 19/06/2019.

${ }^{4}$ Universidade Bandeirante de São Paulo.

${ }^{5}$ Universidade Federal do ABC.

${ }^{6}$ Universidade Federal de Pernambuco.
} 


\begin{tabular}{|c|c|}
\hline TRABALHO & CÓDIGO \\
\hline $\begin{array}{l}\text { Conhecimento do professor de Matemática sobre equações: analisando o } \\
\text { processo avaliativo sob o olhar de um modelo de perfil conceitual. Thais } \\
\text { Helena Inglêz Silva. Dissertação de mestrado acadêmico. } 2015\end{array}$ & D06UFABC2015 \\
\hline $\begin{array}{l}\text { Perfil conceitual de equação: investigações acerca das concepções de alunos } \\
\text { de licenciaturas em Matemática. Marieli Vanessa Rediske de Almeida. } \\
\text { Dissertação de mestrado acadêmico. } 2016 .\end{array}$ & D07UFABC2016 \\
\hline $\begin{array}{l}\text { Conhecimentos de professores para o ensino da leitura e construção de } \\
\text { gráficos estatísticos na Educação Básica. Eduardo Keidin Sera. Dissertação } \\
\text { de mestrado acadêmico. } 2016 \text {. }\end{array}$ & D08UNIAN 72016 \\
\hline $\begin{array}{l}\text { Além da zona de conforto: problematizando o conhecimento de Matemática } \\
\text { para o ensino. Carolina Moura Brasil Carneiro da Silva. Dissertação de } \\
\text { mestrado acadêmico. } 2016 \text {. }\end{array}$ & D09UFRJ ${ }^{8} 2016$ \\
\hline $\begin{array}{l}\text { Formação de professores dos Anos Iniciais: uma investigação sobre os } \\
\text { conhecimentos para o ensino de área e perímetro de figuras planas. Susana } \\
\text { Maris França da Silva. Dissertação de mestrado acadêmico. } 2016 \text {. }\end{array}$ & D10UNIAN2016 \\
\hline $\begin{array}{l}\text { Álgebra nos Anos Iniciais do Ensino Fundamental: uma análise do } \\
\text { conhecimento matemático acerca do Pensamento Algébrico. Miriam Criez } \\
\text { Nobrega Ferreira. Dissertação de mestrado acadêmico. } 2017 \text {. }\end{array}$ & D11UFABC2017 \\
\hline $\begin{array}{l}\text { Conhecimentos matemáticos de professores dos Anos Iniciais do Ensino } \\
\text { Fundamental: um estudo sobre o jogo da velha com figuras geométricas } \\
\text { como recurso didático. Regina de Lima Silva. Dissertação de mestrado } \\
\text { acadêmico. } 2017 \text {. }\end{array}$ & D12UFPE2017 \\
\hline $\begin{array}{l}\text { Aprendendo a ensinar geometria nos Anos Iniciais do Ensino Fundamental: } \\
\text { um estudo com alunos de Pedagogia de uma universidade federal mineira. } \\
\text { Hudney Alves Faria de Carvalho. Dissertação de mestrado profissional. } \\
\text { 2017. }\end{array}$ & D13UFOP $^{9} 2017$ \\
\hline $\begin{array}{l}\text { Reflexões de professores de Matemática sobre funções na avaliação da } \\
\text { aprendizagem em processo. Vera Mônica Ribeiro. Dissertação de mestrado } \\
\text { acadêmico. } 2017 \text {. }\end{array}$ & D14UNIAN2017 \\
\hline $\begin{array}{l}\text { O Conhecimento Matemático para o Ensino e os "por quês" dos alunos. } \\
\text { Rodrigo Donizete Serra. Dissertação de mestrado acadêmico. } 2018 .\end{array}$ & D15UFSCAR ${ }^{10} 2018$ \\
\hline $\begin{array}{l}\text { Conhecimento matemático específico para o ensino na Educação Básica: a } \\
\text { álgebra na escola e na formação do professor. Maria Cristina Costa } \\
\text { Ferreira. Tese de doutorado. 2014. }\end{array}$ & T01UFMG ${ }^{11} 2014$ \\
\hline $\begin{array}{l}\text { Um estudo sobre os conhecimentos necessários ao professor de Matemática } \\
\text { para a exploração de noções concernentes às demonstrações e provas na } \\
\text { Educação Básica. Marta Élid Amorim Mateus. Tese de doutorado. } 2015 .\end{array}$ & T02UNIAN2015 \\
\hline $\begin{array}{l}\text { A base de conhecimento para o ensino de sólidos arquimedianos. Talita } \\
\text { Carvalho Silva de Almeida. Tese de doutorado. } 2015 \text {. }\end{array}$ & T03PUCSP ${ }^{12} 2015$ \\
\hline $\begin{array}{l}\text { Conhecimentos de professores dos anos iniciais para o ensino dos números } \\
\text { racionais em sua representação decimal. Norma Kerches de Oliveira Rogeri. } \\
\text { Tese de doutorado. } 2015 \text {. }\end{array}$ & T04UNIAN2015 \\
\hline $\begin{array}{l}\text { Professor de Matemática em início de carreira: contribuições do Pibid. } \\
\text { Rafael Neves Almeida. Tese de doutorado. } 2015 .\end{array}$ & T05UNIAN2015 \\
\hline
\end{tabular}

\footnotetext{
${ }^{7}$ Universidade Anhanguera de São Paulo.

${ }^{8}$ Universidade Federal do Rio de Janeiro.

${ }^{9}$ Universidade Federal de Ouro Preto.

${ }^{10}$ Universidade Federal de São Carlos.

${ }^{11}$ Universidade Federal de Minas Gerais.

${ }^{12}$ Pontifícia Universidade Católica de São Paulo.
} 


\begin{tabular}{|l|c|}
\hline \multicolumn{1}{|c|}{ TRABALHO } & CÓDIGO \\
\hline $\begin{array}{l}\text { Aprendizagem e constituição profissional de uma professora de Matemática: } \\
\text { um estudo de si. Thaís de Oliveira. Tese de doutorado. 2015. }\end{array}$ & T06UNICAMP ${ }^{132015}$ \\
\hline $\begin{array}{l}\text { Modelagem Matemática e mobilização de conhecimentos didático- } \\
\text { matemáticos na formação continuada de professores dos Anos Iniciais. } \\
\text { Rogério Marques Ribeiro. Tese de doutorado. 2016. }\end{array}$ & T07UFSCAR2016 \\
\hline $\begin{array}{l}\text { Contribuições do campo conceitual multiplicativo para a formação inicial de } \\
\text { professores de Matemática com suporte das tecnologias digitais. Rodrigo } \\
\text { Lacerda Carvalho. Tese de doutorado. 2017. }\end{array}$ & T08UFC ${ }^{14} 2017$ \\
\hline $\begin{array}{l}\text { Conhecimento Matemático para o Ensino de polinômios na Educação } \\
\text { Básica. Etienne Lautenschlager. Tese de doutorado. 2017. }\end{array}$ & T09UFABC2017 \\
\hline $\begin{array}{l}\text { Conhecimento especializado do professor dos anos iniciais no âmbito da } \\
\text { multiplicação: uma metassíntese de teses produzidas entre 2001 e 2012 em } \\
\text { diferentes contextos formativos. Rosana Catarina Rodrigues de Lima. Tese } \\
\text { de doutorado. 2018. }\end{array}$ & T10UNICAMP2018 \\
\hline $\begin{array}{l}\text { Escala apresentada em gráficos: Conhecimentos Matemáticos para o Ensino } \\
\text { dos Anos Iniciais do Ensino Fundamental (crianças e EJA). Milka Rossana } \\
\text { Guerra Cavalcanti de Albuquerque. Tese de doutorado. 2018. }\end{array}$ & T11UFPE2018 \\
\hline
\end{tabular}

Fonte: Da pesquisa.

As análises apresentadas neste artigo foram realizadas a partir de procedimentos de descrição e interpretação dos resumos desses trabalhos, por considerarmos que eles teriam elementos que nos permitiriam atingir o objetivo proposto. Após o levantamento dos trabalhos que seriam analisados, selecionamos seus resumos, codificamos e evidenciamos os objetivos gerais neles apresentados ${ }^{15}$ para, a partir deles, buscarmos relações numa tentativa de agrupálos por suas semelhanças.

Em dissertações ou teses em que os objetivos gerais descritos no resumo não eram suficientes para identificarmos a partir de que elementos o MKT fora investigado, e ou os elementos que foram analisados com base no modelo, recorremos também a trechos do resumo referentes aos percursos metodológicos da pesquisa. Com isso, elaboramos o Quadro 2, que contém os fragmentos dos resumos dos trabalhos que nos permitiram realizar os agrupamentos propostos.

Quadro 2 - Excertos dos resumos das teses e dissertações.

\section{TRECHOS DOS RESUMOS}

"O objetivo dessa pesquisa foi investigar a compreensão e as reflexões dos professores de Matemática relativas a resultados de avaliações externas do SARESP (Sistema de Avaliação de Rendimento Escolar do Estado de São Paulo) em um contexto de formação continuada envolvendo o Ensino de

\footnotetext{
${ }^{13}$ Universidade Estadual de Campinas.

${ }^{14}$ Universidade Federal do Ceará.

${ }^{15}$ O texto completo da tese T09-UFABC2017 não estava disponível nos bancos de dados e na biblioteca digital do programa de pós-graduação da UFABC. Por esse motivo, utilizamos o resumo do trabalho disponível na biblioteca digital da UFABC.
} 


\begin{tabular}{|c|c|}
\hline TRECHOS DOS RESUMOS & TRABALHO \\
\hline \multicolumn{2}{|l|}{$\begin{array}{l}\text { Álgebra na Educação Básica, em particular o de equações e sistemas de } \\
\text { equações." }\end{array}$} \\
\hline $\begin{array}{l}\text { "Os objetivos dessa pesquisa consistem em mapear, investigar e compreender } \\
\text { quais os conhecimentos algébricos que são mobilizados por professores } \\
\text { quando estão elaborando suas aulas sobre equaçoses para a Educação Básica." }\end{array}$ & D02UFABC2014 \\
\hline $\begin{array}{l}\text { "A presente dissertação teve por objetivo investigar como os professores dos } \\
\text { anos iniciais do Ensino Fundamental julgam propostas de ensino para o } \\
\text { trabalho com os números racionais, tomando por base as expectativas de } \\
\text { aprendizagem dos Parâmetros Curriculares de Matemática de Pernambuco." }\end{array}$ & D03UFPE2015 \\
\hline $\begin{array}{l}\text { "[...] busca compreender como o conceito de simetria se manifesta na álgebra } \\
\text { escolar por meio da análise de documentos oficiais e manuais do professor." }\end{array}$ & D04UFABC2015 \\
\hline $\begin{array}{l}\text { "[...] investigou como se apresenta o conhecimento pedagógico dos números } \\
\text { racionais com professores de Matemática durante a sua prática em parceria } \\
\text { com o projeto do Observatório da Educação da UFABC [...]" }\end{array}$ & D05UFABC2015 \\
\hline $\begin{array}{l}\text { "Intenta-se compreender quais conhecimentos sobre o ensino de equação } \\
\text { professores dos anos finais do Ensino Fundamental e do Ensino Médio } \\
\text { manifestam, particularmente ao refletirem sobre seus próprios processos } \\
\text { avaliativos." }\end{array}$ & D06UFABC2015 \\
\hline $\begin{array}{l}\text { "[...] essa pesquisa teve por objetivo identificar e compreender as concepções } \\
\text { de equação de licenciandos em Matemática." }\end{array}$ & D07UFABC2016 \\
\hline $\begin{array}{l}\text { "A finalidade desse estudo foi investigar a ampliação da base de } \\
\text { conhecimentos de um grupo de professores de Matemática da Educação } \\
\text { Básica para ensinar leitura e construção de gráficos estatísticos a alunos dos } \\
\text { anos finais do Ensino Fundamental e do Médio, mediante uma formação } \\
\text { continuada, cujos pressupostos foram reflexões compartilhadas sobre o } \\
\text { Letramento Estatístico e dificuldades de ensinar noções relativas ao tema." }\end{array}$ & D08UNIAN2016 \\
\hline $\begin{array}{l}\text { "O presente trabalho tem por objetivo apresentar e analisar uma tarefa } \\
\text { desenhada com o intuito de explorar o conhecimento matemático para o ensino } \\
\text { de professores em formação continuada, por meio de suas próprias reflexões } \\
\text { sobre conteúdos chave do ensino básico como, por exemplo, a divisão de } \\
\text { números naturais." }\end{array}$ & D09UFRJ2016 \\
\hline $\begin{array}{l}\text { "A finalidade desse estudo foi investigar, durante sessões de estudos de } \\
\text { professoras que lecionam Matemática para os anos iniciais de uma escola } \\
\text { particular da grande São Paulo, o desenvolvimento do conhecimento } \\
\text { profissional docente sobre os conceitos de área e perímetro e seu ensino." }\end{array}$ & D10UNIAN2016 \\
\hline $\begin{array}{l}\text { "O objetivo deste trabalho foi investigar o conhecimento matemático para o } \\
\text { ensino do Pensamento Algébrico nos Anos Iniciais do Ensino Fundamental. } \\
\text { [...] a partir do curso de formação oferecido buscamos identificar os } \\
\text { conhecimentos matemáticos revelados pelo grupo de professores quando estes } \\
\text { discutem tarefas com potencial algébrico." }\end{array}$ & D11UFABC2017 \\
\hline $\begin{array}{l}\text { "Esta pesquisa teve por objetivo analisar os conhecimentos matemáticos } \\
\text { mobilizados por professores ao lidarem com o Jogo da Velha com Figuras } \\
\text { Geométricas como recurso didático." }\end{array}$ & D12UFPE2017 \\
\hline $\begin{array}{l}\text { "A presente pesquisa teve como propósito analisar possíveis mobilizações de } \\
\text { saberes por estudantes de Pedagogia, ao vivenciarem tarefas relacionadas ao } \\
\text { ensino de Geometria nos anos iniciais do Ensino Fundamental [...]" }\end{array}$ & D13UFOP2017 \\
\hline $\begin{array}{l}\text { "O objetivo da pesquisa foi o de analisar, ao longo de um processo formativo, } \\
\text { a compreensão evidenciada por professores de Matemática sobre a Avaliação } \\
\text { da Aprendizagem em Processo (AAP), no tocante às questões que envolvem } \\
\text { o conteúdo de função." }\end{array}$ & D14UNIAN2017 \\
\hline $\begin{array}{l}\text { "Tem como objetivos compreender quais conhecimentos para o ensino de } \\
\text { Matemática são mobilizados a partir da reflexão sobre os por quês dos }\end{array}$ & D15UFSCAR2018 \\
\hline
\end{tabular}




\begin{tabular}{|c|c|}
\hline $\begin{array}{l}\text { TRECHOS DOS RESUMOS } \\
\end{array}$ & TRABALHO \\
\hline $\begin{array}{l}\text { alunos, esclarecer conceitualmente o que compõe o conhecimento para o } \\
\text { ensino de Matemática e revelar, nos Por Quês dos alunos, potencialidades } \\
\text { formativas para os professores que ensinam Matemática." }\end{array}$ & \\
\hline $\begin{array}{l}\text { "O objetivo desta pesquisa é identificar elementos constituintes desse } \\
\text { conhecimento matemático específico do professor, no que se refere } \\
\text { particularmente ao trabalho com a álgebra na Educação Básica." }\end{array}$ & T01UFMG2014 \\
\hline $\begin{array}{l}\text { "Esta pesquisa teve o propósito de refletir sobre o tipo de formação inicial que } \\
\text { um futuro professor de Matemática deveria vivenciar para a seleção, } \\
\text { organização e elaboração de situações que favoreçam a aprendizagem de seus } \\
\text { alunos da Educação Básica de ideias fundamentais relativas às demonstrações } \\
\text { e provas." }\end{array}$ & T02UNIAN2015 \\
\hline $\begin{array}{l}\text { "O presente trabalho tem como objetivo identificar os saberes docentes } \\
\text { mobilizados para que sólidos arquimedianos sejam ensinados. Assim, a } \\
\text { pergunta de pesquisa foi: qual base de conhecimento para o ensino de sólidos } \\
\text { arquimedianos na escola básica? Para responder a esta questão, recorremos a } \\
\text { um estudo bibliográfico desenvolvido com base em material já elaborado, } \\
\text { constituídos principalmente de artigos científicos." }\end{array}$ & T03PUCSP2015 \\
\hline $\begin{array}{l}\text { "A finalidade desse estudo foi investigar a ampliação da base de } \\
\text { conhecimentos de um grupo de professores dos anos iniciais do Ensino } \\
\text { Fundamental para ensinar números racionais, especialmente a representação } \\
\text { decimal, mediante uma formação continuada, cujos pressupostos foram } \\
\text { reflexões compartilhadas sobre práticas docentes e sobre dificuldades de } \\
\text { aprendizagem de noções relativas a esse tema por alunos da Educação Básica." }\end{array}$ & T04UNIAN2015 \\
\hline $\begin{array}{l}\text { "Este trabalho teve por objetivo estudar contribuições do Programa } \\
\text { Institucional de Bolsas de Iniciação à Docência - Pibid, da Licenciatura da } \\
\text { Universidade Federal de Sergipe, campus Itabaiana, para a prática docente de } \\
\text { professores de Matemática." }\end{array}$ & T05UNIAN2015 \\
\hline $\begin{array}{l}\text { "Esta pesquisa tem por objetivo compreender como as experiências de } \\
\text { aprendizagem de matemática e de educação matemática da pesquisadora, } \\
\text { enquanto estudante e professora de matemática, vêm influenciando sua prática } \\
\text { de ensinar matemática e sua constituição profissional, sobretudo em relação a } \\
\text { seus conhecimentos, suas crenças e sua identidade." }\end{array}$ & T06UNICAMP2015 \\
\hline $\begin{array}{l}\text { "Este estudo teve como objetivo investigar a formação continuada de } \\
\text { professores que ensinam matemática nos anos iniciais do Ensino Fundamental } \\
\text { I, considerando um ambiente de aprendizagem na perspectiva da Modelagem } \\
\text { Matemática. Buscou-se investigar como esta formação continuada poderia } \\
\text { contribuir para reflexões acerca do conhecimento para se ensinar matemática } \\
\text { nesse nível de ensino." }\end{array}$ & T07UFSCAR2016 \\
\hline $\begin{array}{l}\text { "[...] nosso objetivo geral é analisar o processo de construção dos conceitos de } \\
\text { estruturas multiplicativas e de função, por parte de futuros professores de } \\
\text { Matemática, nos domínios conceitual e pedagógico, a partir do uso de } \\
\text { tecnologias digitais." }\end{array}$ & T08UFC2017 \\
\hline $\begin{array}{l}\text { "[...] investigar se e como o conceito anel de polinômios é (re)construído por } \\
\text { professores de Matemática que lecionam na Educação Básica [...]. Para a } \\
\text { produção de dados da pesquisa, utilizou-se como instrumentos metodológicos } \\
\text { os questionários e os registros escritos, elaborados/produzidos pelos } \\
\text { professores-participantes da pesquisa" }\end{array}$ & T09UFABC2017 \\
\hline $\begin{array}{l}\text { "Assim esta pesquisa buscou investigar a abordagem dada por pesquisas } \\
\text { acadêmicas brasileiras ao conhecimento especializado do professor que ensina } \\
\text { matemática (PEM) nos anos iniciais de escolarização em relação à } \\
\text { multiplicação [...]" }\end{array}$ & T10UNICAMP2018 \\
\hline
\end{tabular}




\section{TRECHOS DOS RESUMOS}

"O objetivo desse estudo foi estabelecer Conhecimentos Matemáticos para o Ensino de escala apresentada em gráficos estatísticos necessários para desenvolver um processo de formação continuada de professores dos anos

T11UFPE2018 iniciais do Ensino Fundamental."

$$
\text { Fonte: Da pesquisa }
$$

\section{Resultados}

A partir da leitura dos resumos dos trabalhos e das semelhanças encontradas nas informações presentes, principalmente, nos excertos selecionados, obtivemos cinco agrupamentos, nomeadamente: reflexões, compreensões, concepções; ações formativas; práticas profissionais; documentos oficiais e pesquisas bibliográficas, que constituem o Quadro 3.

Quadro 3 - Agrupamentos de dissertações e teses brasileiras que utilizaram o MKT.

\begin{tabular}{|c|c|}
\hline $\begin{array}{c}\text { Elementos a partir dos quais o MKT } \\
\text { tem sido investigado e ou que foram } \\
\text { analisados com base no modelo }\end{array}$ & DISSERTAÇõES E TESES \\
\hline \multirow{2}{*}{ Reflexões, compreensões, concepções } & D09UFRJ2016; D06UFABC2015; D15UFSCAR2018; \\
& $\begin{array}{c}\text { D07UFABC2016; D08UNIAN2016; D01UNIBAN2012; } \\
\text { D14UNIAN2017; T04UNIAN2015; T09UFABC2017. }\end{array}$ \\
\hline & D11UFABC2017; D02UFABC2014; D10UNIAN2016; \\
Ações formativas & D13UFOP2017; T02UNIAN2015; T07UFSCAR2016; \\
& T11UFPE2018; T08UFC2017. \\
\hline Práticas profissionais & D05UFABC2015; D12UFPE2017; T06UNICAMP2015; \\
\hline Documentos oficiais & T01UFMG2014; T05UNIAN2015. \\
\hline Pesquisas bibliográficas & D04UFABC2015; D03UFPE2015. \\
\hline & T03PUCSP2015; T10UNICAMP2018. \\
\hline
\end{tabular}

Fonte: Da pesquisa

Feita a constituição dos agrupamentos, realizamos interpretações e algumas inferências, buscando dialogar com a literatura e construir compreensões a respeito do assunto abordado.

Segundo Alarcão,

[...] os professores desempenham um importante papel na produção e estruturação do conhecimento pedagógico porque refletem, de uma forma situada, na e sobre a interação que se gera entre o conhecimento científico [...] e a sua aquisição pelo aluno, refletem na e sobre a interação entre a pessoa do professor e a pessoa do aluno, entre a instituição escola e a sociedade em geral. Desta forma, têm um papel ativo na educação e não um papel meramente técnico que se reduza à execução de normas e receitas ou à aplicação de teorias exteriores à sua própria comunidade profissional (2005, p. 176).

Desse modo, as reflexões de professores e futuros professores sobre conteúdos e práticas de ensino podem fazer com que eles manifestem aspectos de seus conhecimentos profissionais e modifiquem e ampliem sua base de conhecimentos. 
Assim como as reflexões, suas compreensões e concepções também influenciam seus conhecimentos profissionais. Curi e Santos (2011, p. 3), por exemplo, destacam que "as concepções que os professores têm sobre Matemática e seu ensino interferem na constituição de seus conhecimentos".

Isso pode ser corroborado pelos trabalhos que investigaram o MKT a partir de, ou utilizaram o Conhecimento Matemático para o Ensino para analisar reflexões, compreensões, concepções dos participantes da pesquisa. Com base nos excertos analisados, inferimos que tais elementos oportunizaram a identificação e ou análise dos conhecimentos nas dissertações e teses que o investigaram em processos de formação inicial e continuada, seja acerca de um ou mais conceitos ou conteúdos (D09UFRJ2016, D07UFABC2016, T04UNIAN2015, T09UFABC2017), de aspectos relativos ao ensino e à aprendizagem, como os porquês dos alunos (D15UFSCAR2018) e o Letramento Estatístico (D08UNIAN2016), seja acerca de processos avaliativos (D06UFABC2015) ou de avaliações externas (D01UNIBAN2012, D14UNIAN2017).

Ainda com relação à reflexão, consideramos que os professores, ao refletirem, estão em processos que "promovem a tomada de consciência dos processos de aprendizagem; revelam o caráter formativo de algumas práticas de sala de aula" (PASSOS et al., 2006, p. 202) e, além disso, ampliam e enriquecem sua aprendizagem profissional, sendo este último aspecto também destacado por autores como Ponte et al. (2017, p. 73):

\section{Tal como os alunos aprendem Matemática trabalhando em tarefas matemáticas e argumentando com os seus colegas ou refletindo sobre os seus raciocínios e os seus resultados, também os professores fazem as suas aprendizagens profissionais sobretudo a partir da sua atividade e da reflexão sobre a sua atividade realizada em contextos tanto quanto possível próximos da sua prática profissional.}

Esses contextos mencionados por Ponte et al. (2017) são compostos de ações formativas que abordam necessidades e potencialidades do professor. Dentre essas ações, podemos apontar, por exemplo, aquelas desenvolvidas nas dissertações e teses que compõem o agrupamento que constituímos com esse título, quais sejam: discussões em grupo a respeito de tarefas com potencial algébrico (D11UFABC2017), o planejamento de uma aula (D02UFABC2014), o estudo para o desenvolvimento de um curso de formação continuada (T11UFPE2018), estudos de temas cuja necessidade é comum a um grupo de professores (D10UNIAN2016), ou mesmo situações em que os sujeitos vivenciem tarefas e o ensino de determinados assuntos em seus cursos de formação inicial (D13UFOP2017, T02UNIAN2015, T08UFC2017) ou uma metodologia específica em propostas de formação continuada (T07UFSCAR2016). 
Em todos esses trabalhos, encontramos a semelhança de o MKT ter sido abordado a partir de ações formativas que oportunizam ao professor ou futuro professor estar em constante aprendizado, o que pode auxiliá-lo em sua prática profissional que se dá em diferentes contextos, sendo um deles o PIBID (Programa Institucional de Bolsa de Iniciação à Docência), que oportuniza ao futuro professor interação com docentes experientes em contextos educativos, favorecendo sua aprendizagem (NASCIMENTO; CASTRO; LIMA, 2017). As contribuições do programa da Licenciatura da Universidade Federal de Sergipe, campus Itabaiana, para a prática docente de professores de Matemática foram investigadas em um dos trabalhos (T05UNIAN2015) do agrupamento práticas profissionais a partir do que vivenciaram os ex-bolsistas.

Nesse agrupamento, também consideramos os textos em que o Conhecimento Matemático para o Ensino foi investigado a partir do trabalho realizado por professores da Educação Básica no ensino de álgebra (T01UFMG2014), a partir do trabalho com números racionais (D05UFABC2015), e com jogos em sala de aula (D12UFPE2017). A diversidade de práticas profissionais presentes nas investigações a respeito dos conhecimentos aponta para a ideia de que eles são específicos da profissão e mobilizados em diferentes situações da prática (PONTE; OLIVEIRA, 2002).

Nesse sentido também, em um dos trabalhos do agrupamento (T06UNICAMP2015), a autora busca compreender como suas experiências com a Matemática e a Educação Matemática, enquanto professora e enquanto estudante, vêm influenciando sua prática profissional e a constituição de seus conhecimentos.

Além de aspectos relacionados à experiência prática, autores como Flores-Mendrano et al. (2014) têm trazido os resultados de pesquisas em Educação Matemática como um elemento do conhecimento profissional necessário ao professor de Matemática. No conjunto de textos considerados para análise, identificamos dois trabalhos (T03PUCSP2015 e T10UNICAMP2018), que compõem o agrupamento dos que investigaram o MKT a partir de pesquisas bibliográficas, e outros dois (D04UFABC2015 e D03UFPE2015), que compõem o agrupamento de pesquisas que utilizaram o MKT para analisar, ou investigaram o modelo, a partir de documentos oficiais, itens que se configuram como importantes para o professor conhecer, visto que esses documentos apresentam, entre outros aspectos, o currículo e "é conveniente para um professor conhecer em profundidade e integrar em seu trabalho cada um dos aspectos contemplados no currículo.” (FLORES-MENDRANO et al., 2014, p. 84) 


\section{Algumas CONSIDERAÇõeS}

Neste trabalho tivemos como objetivo identificar a partir de quais elementos o Conhecimento Matemático para o Ensino tem sido utilizado e ou identificar os elementos que têm sido analisados com base no MKT em dissertações e teses brasileiras. Com base no levantamento realizado, foi possível destacar cinco agrupamentos contendo tais elementos: reflexões, compreensões, concepções; ações formativas; práticas profissionais; pesquisas bibliográficas e documentos oficiais.

A identificação de elementos por meio dos quais se tem investigado o MKT ou que foram analisados a partir do modelo, realizada neste trabalho, pode auxiliar formadores de professores na proposição de ações com o objetivo de desenvolver ou mobilizar conhecimentos profissionais de professores e futuros professores de Matemática. Os trabalhos do agrupamento ações formativas, por exemplo, podem ser utilizados como fonte de busca pelos formadores para o planejamento de tais ações. Além disso, essa identificação pode orientar os pesquisadores da área para pesquisas futuras, promovendo ideias e discussões de modo a contribuir para a Educação Matemática.

Nesse sentido, ressaltamos a importância de que haja mais teses e dissertações a respeito do conhecimento matemático para o ensino na formação inicial, explorando suas diversas possibilidades tendo em vista o desenvolvimento profissional do futuro professor, dado que poucos trabalhos do conjunto de textos analisados abordam esse contexto.

Como sugestão de pesquisas futuras, destacamos a possibilidade de analisar os resultados dos trabalhos aqui apresentados, bem como identificar o que tem sido investigado sobre outros modelos de conhecimentos profissionais dos professores de Matemática.

\section{REFERÊNCIAS}

ALARCÃO, Isabel. (Coord.). Formação reflexiva de professores: estratégias de supervisão. Porto: Porto Editora, 2005.

ALBUQUERQUE, Milka Rossana Guerra Cavalcanti de. Escala apresentada em gráficos: conhecimentos matemáticos para o ensino dos anos iniciais do ensino fundamental (crianças e Eja). 2019. Tese (Doutorado em Educação Matemática e Tecnológica) Universidade Federal de Pernambuco, Recife, PE, 2019.

ALMEIDA, Talita Carvalho Silva de. A base de conhecimento para o ensino de sólidos arquimedianos. 2015. Tese (Doutorado em Educação Matemática) - Pontifícia Universidade Católica de São Paulo, São Paulo, SP, 2015. Disponível em:

https://sucupira.capes.gov.br/sucupira/public/consultas/coleta/trabalhoConclusao/viewTrabalh oConclusao.jsf?popup=true\&id_trabalho=3141211. Acesso em: 08 mai. 2020. 
ALMEIDA, Marieli Vanessa Rediske de. Perfil conceitual de equação: investigações acerca das concepções de alunos de licenciaturas em matemática. 2016. Dissertação (Mestrado em Ensino, História e Filosofia das Ciências e Matemática) - Universidade Federal do ABC, Santo André, SP, 2016. Disponível em:

https://sucupira.capes.gov.br/sucupira/public/consultas/coleta/trabalhoConclusao/viewTrabalh oConclusao.jsf?popup=true\&id_trabalho=3608591. Acesso em: 08 mai. 2020.

ALMEIDA, Rafael Neves. Professor de matemática em início de carreira: contribuições do PIBID. 2016. Tese (Programa de Doutorado em Educação Matemática) - Universidade Anhanguera de São Paulo, São Paulo, SP, 2016. Disponível em:

https://sucupira.capes.gov.br/sucupira/public/consultas/coleta/trabalhoConclusao/viewTrabalh oConclusao.jsf?popup=true\&id_trabalho=3023257. Acesso em: 08 mai. 2020.

ANDO, Rosangela de Souza Jorge. Formação Continuada e Ensino de Álgebra: Reflexões de Professores da Educação Básica sobre itens do Saresp. 2012. Dissertação (Mestrado em Educação Matemática) - Universidade Anhanguera de São Paulo, São Paulo, SP, 2012. Disponível em: https://repositorio.pgsskroton.com.br/handle/123456789/3656? mode=full. Acesso em: 08 mai. 2020.

BALL, Deborah Lowenberg; THAMES, Mark Hoover; PHELPS, Geoffrey. Content Knowledge for teaching: what makes it special? Journal of Teacher Education, v.59, n.5, p.389-407, 2008.

BOGDAN, Robert, BIKLEN, Sari. Investigação qualitativa em educação. Tradução Maria João Alvarez, Sara Bahia dos Santos e Telmo Mourinho Baptista. Porto: Porto Editora, 1994.

CARVALHO, Hudney Alves Faria de. Aprendendo a ensinar geometria nos anos iniciais do Ensino Fundamental: um estudo com alunos de pedagogia de uma Universidade Federal mineira. 2017. Dissertação (Mestrado em Educação Matemática) - Instituto de ciências Exatas e Biológicas, Universidade Federal de Ouro Preto, Ouro Preto, MG, 2017. Disponível em:

https://sucupira.capes.gov.br/sucupira/public/consultas/coleta/trabalhoConclusao/viewTrabalh oConclusao.jsf?popup=true\&id_trabalho=5402983. Acesso em: 08 mai. 2020.

CARVALHO, Rodrigo Lacerda. Contribuições do campo conceitual multiplicativo para a formação inicial de professores de Matemática com suporte das tecnologias digitais. 2017. Tese (Doutorado em Educação) - Universidade Federal do Ceará - Faculdade de Educação, Fortaleza, CE, 2017. Disponível em:

https://sucupira.capes.gov.br/sucupira/public/consultas/coleta/trabalhoConclusao/viewTrabalh oConclusao.jsf?popup=true\&id trabalho=4990969. Acesso em: 8 mai. 2020.

CURI, Edda; SANTOS, Cintia Aparecida Bento dos. Algumas reflexões sobre o tratamento de conteúdos do ensino básico em um curso de licenciatura em matemática. EM TEIA -

Revista de Educação Matemática e Tecnológica Iberoamericana, v. 2, n. 2, p. 1-19, 2011. Disponível em: https://periodicos.ufpe.br/revistas/emteia/article/viewFile/2154/1723. Acesso em: 08 mai. 2020.

FERREIRA, Maria Cristina Costa. Conhecimento matemático específico para o ensino na Educação Básica. 2014. Tese (Doutorado) - Universidade Federal de Minas Gerais Faculdade de Educação, Programa de Pós-Graduação em Educação: conhecimento e inclusão 
social, Belo Horizonte, MG, 2014. Disponível em: https://repositorio.ufmg.br/handle/1843/BUOS-9PMKNE. Acesso em: 09 mai. 2020.

FERREIRA, Miriam Criez Nobrega. Álgebra nos Anos Iniciais do Ensino Fundamental: uma análise do conhecimento matemático acerca do Pensamento Algébrico. 2017. Dissertação (Mestrado em Ensino, História e Filosofia das Ciências e Matemática) Universidade Federal do ABC, Santo André, SP, 2017. Disponível em: http://biblioteca.ufabc.edu.br/. Acesso em: 08 mai. 2020.

FLORES-MENDRANO, Eric; ESCUDERO-ÁVILA, Dinazar; MONTES, Miguel; AGUILAR, Álvaro; CARRILLO, José. (2014). Nuestra modelación del conocimiento especializado del profesor de matemáticas, el MTSK. In: AGUILAR, Álvaro et al. Un marco teórico para el conocimiento especializado del profesor de matemáticas. Huelva, España: Universidad de Huelva Publicaciones, 2014, p. 57-72.

GARCIA, Tânia Marli Rocha. Identidade Profissional de Professores de Matemática em uma Comunidade de Prática. 2014. Tese (Doutorado em Ensino de Ciências e Educação Matemática) - Universidade Estadual de Londrina, Londrina, 2014. Disponível em: http://www.uel.br/pos/mecem/arquivos_pdf/2014TESETANIAGARCIA.pdf. Acesso em: 08 mai. 2020.

LAUTENSCHLAGER, Etienne. Conhecimento matemático para o ensino de polinômios na educação básica. 2017. Tese de doutorado (Doutorado em Neurociência e cognição) Universidade Federal do ABC, Santo André, SP, 2017.

LIMA, Rosana Catarina Rodrigues de. Conhecimento especializado do professor dos anos iniciais no âmbito da multiplicação: Uma metassíntese de teses produzidas entre 2001 e 2012 em diferentes contextos formativos. 2018. Tese (Doutorado) - Faculdade de Educação, Universidade Estadual de Campinas, Campinas, SP, 2018. Disponível em: http://repositorio.unicamp.br/bitstream/REPOSIP/331852/1/Lima_RosanaCatarinaRodrigues De_D.pdf. Acesso em: 08 mai. 2020.

MATEUS, Marta Élid Amorim. Um estudo sobre os conhecimentos necessários ao professor de Matemática para a exploração de noções concernentes às demonstrações e provas na Educação Básica. 2015. Tese (Doutorado em Educação Matemática) Universidade Anhanguera de São Paulo, São Paulo, SP, 2015. Disponível em: https://sucupira.capes.gov.br/sucupira/public/consultas/coleta/trabalhoConclusao/viewTrabalh oConclusao.jsf?popup=true\&id_trabalho=2356651. Acesso em: 08 mai. 2020.

MORIEL JUNIOR, Jeferson Gomes; WIELEWSKI, Gladys Denise. Base de Conhecimento de Professores de Matemática: do Genérico ao Especializado. Revista de Ensino, Educação e Ciências Humanas. v.18, n.2, p.126-133, 2017. Disponível em: https://www.researchgate.net/publication/318702674_Base_de_conhecimento_de_professores _de_matematica_do_generico_ao_especializado. Acesso em: 08 mai. 2020.

NASCIMENTO, Francisco Jeovane do; CASTRO, Eliziane Rocha; LIMA, Ivoneide Pinheiro de. Desenvolvimento profissional de professores de matemática iniciantes: contribuição do PIBID. Revista Eletrônica de Educação, v.11, n.2, p.487-504, jun./ago., 2017. Disponível em: http://www.reveduc.ufscar.br/index.php/reveduc/article/view/1962/609. Acesso em: 08 mai. 2020. 
OLIVEIRA, Bárbara Passadore de. O conceito de simetria na álgebra escolar: um estudo baseado na análise de documentos oficiais e manuais de professores. 2015. Dissertação (Mestrado) - Universidade Federal do ABC, Santo André, SP, 2015. Disponível em: http://biblioteca.ufabc.edu.br/index.html. Acesso em: 08 mai. 2020.

OLIVEIRA, Felipe Augusto Pereira Vasconcelos Santos e. Analisando a mobilização de conhecimentos algébricos de professores de Educação Básica: $O$ momento de preparação de aulas sobre equações. 2014. Dissertação (Mestrado em Ensino, História e Filosofia das Ciências e Matemática.) - Universidade Federal do ABC. Santo André, SP, 2014. Disponível em:

https://sucupira.capes.gov.br/sucupira/public/consultas/coleta/trabalhoConclusao/viewTrabalh oConclusao.jsf?popup=true\&id_trabalho=1310200. Acesso em: 08 mai. 2020.

OLIVEIRA, Thaís de. Aprendizagem e constituição profissional de uma professora de Matemática: um estudo de si. 2015. Tese (Doutorado) - Instituto de Física Gleb Wataghin Universidade Estadual de Campinas, Campinas, SP, 2015. Disponível em:

https://sucupira.capes.gov.br/sucupira/public/consultas/coleta/trabalhoConclusao/viewTrabalh oConclusao.jsf?popup=true\&id_trabalho=2813860. Acesso em: 08 mai. 2020.

PASSOS, Cármen Lúcia Brancaglion; NACARATO, Adair Mendes; FIORENTINI, Dario; MISKULIN, Rosana Giaretta Sguerra; GRANDO, Regina Célia; GAMA, Renata Prenstteter; MEGID, Maria Auxiliadora Bueno Andrade; FREITAS, Maria Tereza Menezes; MELO, Marisol Vieira de. Desenvolvimento profissional do professor que ensina Matemática: Uma meta-análise de estudos brasileiros. Quadrante, v.XV, n.1 e 2, 2006. Disponível em: https://quadrante.apm.pt/index.php/quadrante/article/view/192. Acesso em: 08 mai. 2020.

PONTE, João Pedro da, OLIVEIRA, Hélia. Remar contra a maré: A construção do conhecimento e da identidade profissional na formação inicial. Revista de Educação, 11(2), p.145-163, 2002. Disponível em:

https://www.researchgate.net/publication/277034808_Remar_contra_a_mare_A_construcao do_conhecimento_e_da_identidade_profissional_na_formacao_inicial. Acesso em: 08 mai. 2020.

PONTE João Pedro da, MATA-PEREIRA, Joana, QUARESMA, Maria, VELEZ, Isabel. Formação de professores dos primeiros anos em articulação com o contexto de prática de ensino de matemática. Revista Latinoamericana de Investigación en Matemática

Educativa, 20 (1), p. 71-94, 2017. Disponível em: http://dx.doi.org/10.12802/relime.17.2013. Acesso em: 08 mai. 2020.

RIBEIRO, Rogério Marques. Modelagem matemática e mobilização de conhecimentos didático-matemáticos na formação continuada de professores dos anos iniciais. 2016. Tese (Doutorado) - Universidade Federal de São Carlos, São Carlos, SP, 2016. Disponível em:

https://sucupira.capes.gov.br/sucupira/public/consultas/coleta/trabalhoConclusao/viewTrabalh oConclusao.jsf?popup=true\&id_trabalho=4259639. Acesso em: 08 mai. 2020.

RIBEIRO, Vera Mônica. Reflexões de professores de matemática sobre funções na avaliação da aprendizagem em processo. 2017. Tese (Doutorado em Educação Matemática) - Universidade Anhanguera de São Paulo, São Paulo, SP, 2017. Disponível em: 
https://sucupira.capes.gov.br/sucupira/public/consultas/coleta/trabalhoConclusao/viewTrabalh oConclusao.jsf?popup=true\&id_trabalho=5822208. Acesso em: 08 mai. 2020.

ROGERI, Norma Kerches de Oliveira. Conhecimentos de professores dos anos iniciais para o ensino dos números racionais em sua representação decimal. 2015. Tese

(Doutorado em Educação Matemática) - Universidade Anhanguera de São Paulo, São Paulo, SP, 2015. Disponível em:

https://sucupira.capes.gov.br/sucupira/public/consultas/coleta/trabalhoConclusao/viewTrabalh oConclusao.jsf?popup=true\&id_trabalho=2759233. Acesso em: 08 mai. 2020.

SANTOS FILHO, Josué Ferreira dos. Investigando como professores dos anos iniciais julgam propostas de ensino para o trabalho com números. 2015. Dissertação (Mestrado em Educação Matemática e Tecnológica) - Universidade Federal de Pernambuco, Recife, PE, 2015. Disponível em:

https://sucupira.capes.gov.br/sucupira/public/consultas/coleta/trabalhoConclusao/viewTrabalh oConclusao.jsf?popup=true\&id_trabalho=2353929. Acesso em: 08 mai. 2020.

SERA, Eduardo Keidin. Conhecimentos de professores para o ensino da leitura e construção de gráficos estatísticos na educação básica. 2016. Dissertação (Mestrado em Educação Matemática) - Universidade Anhanguera de São Paulo, São Paulo, SP, 2016. Disponível em:

https://sucupira.capes.gov.br/sucupira/public/consultas/coleta/trabalhoConclusao/viewTrabalh oConclusao.jsf?popup=true\&id_trabalho=3815938. Acesso em: 08 mai. 2020.

SERRA, Rodrigo Donizete. O Conhecimento Matemático para o Ensino e os "Por Quês" dos alunos. 2018. Dissertação (Programa de Mestrado em Educação) - Universidade Federal de São Carlos, Sorocaba, SP, 2018. Disponível em:

https://sucupira.capes.gov.br/sucupira/public/consultas/coleta/trabalhoConclusao/viewTrabalh oConclusao.jsf?popup=true\&id_trabalho=6335212. Acesso em: 08 mai. 2020.

SHULMAN, Lee. Those who understand: knowledge growth in teaching. Educational Researcher, Washington, v.15, n.2, p.4-14, 1986.

SILVA, Carolina Moura Brasil Carneiro da. Além da Zona de Conforto: Problematizando o Conhecimento de Matemática para o Ensino. 2016. Dissertação (Mestrado em Ensino de Matemática) - Universidade Federal do Rio de Janeiro, Rio de Janeiro, RJ, 2016.

SILVA, Regina de Lima. Conhecimentos matemáticos de professores dos anos iniciais do ensino fundamental: um estudo sobre o jogo da velha com figuras geométricas como recurso didático. 2017. Dissertação (Mestrado em Educação Matemática e Tecnológica) Universidade Federal de Pernambuco, Recife, PE, 2017. Disponível em:

https://sucupira.capes.gov.br/sucupira/public/consultas/coleta/trabalhoConclusao/viewTrabalh oConclusao.jsf?popup=true\&id_trabalho=5730163. Acesso em: 08 mai. 2020.

SILVA, Susana Maris França da. Formação de professor dos anos iniciais: uma investigação sobre os conhecimentos para o ensino de área e perímetro de figuras planas. 2016. Dissertação (Mestrado em Educação Matemática) - Universidade Anhanguera de São Paulo, São Paulo, SP, 2016. Disponível em:

https://sucupira.capes.gov.br/sucupira/public/consultas/coleta/trabalhoConclusao/viewTrabalh oConclusao.jsf?popup=true\&id_trabalho=3817214. Acesso em: 08 mai. 2020. 
SILVA, Thais Helena Inglêz. Conhecimento do professor de Matemática sobre equações: analisando o processo avaliativo sob o olhar de um modelo de perfil conceitual. 2016. Dissertação (Mestrado em Ensino, História e Filosofia das Ciências e Matemática) Universidade Federal do ABC, São Paulo, SP, 2016. Disponível em:

https://sucupira.capes.gov.br/sucupira/public/consultas/coleta/trabalhoConclusao/viewTrabalh oConclusao.jsf?popup=true\&id_trabalho=3104240. Acesso em: 08 mai. 2020.

SOUZA, Debora da Silva. A formação do professor de Matemática: um estudo sobre o conhecimento pedagógico dos números racionais. 2015. Dissertação (Mestrado em Ensino, História e Filosofia das Ciências e Matemática) - Universidade Federal do ABC, Santo André, SP, 2015. Disponível em:

https://sucupira.capes.gov.br/sucupira/public/consultas/coleta/trabalhoConclusao/viewTrabalh oConclusao.jsf?popup=true\&id_trabalho=2464406. Acesso em: 08 mai. 2020.

Recebido em: 13 de maio de 2020.

Aprovado em: 17 de agosto de 2020. 